\title{
Chronic bronchitis is an independently associated factor for more symptom and high-risk groups
}

\author{
This article was published in the following Dove Press journal: \\ International Journal of COPD \\ 21 June 2016 \\ Number of times this article has been viewed
}

Joon Young Choi,' Hyoung Kyu Yoon, ${ }^{2}$ Seoung Ju Park, ${ }^{3}$ Yong Bum Park, ${ }^{4}$ KyeongCheol Shin, ${ }^{5}$ Ju Ock Na, ${ }^{6}$ Kwang $\mathrm{Ha} \mathrm{Yoo,}{ }^{7} \mathrm{Ki}$-Suck Jung, ${ }^{8}$ Young Kyoon Kim,' Chin Kook Rhee'

'Division of Pulmonary, Allergy and Critical Care Medicine, Department of Internal Medicine, Seoul St Mary's Hospital, ${ }^{2}$ Division of Pulmonary and Critical Care Medicine, Department of Internal Medicine, Yeouido St Mary's Hospital, College of Medicine, The Catholic University of Korea, Seoul, ${ }^{3}$ Division of Pulmonary, Allergy, and Critical Care Medicine, Department of Internal Medicine, Chonbuk National University Medical School, Jeonju, ${ }^{4}$ Division of Pulmonary, Allergy, and Critical Care Medicine, Department of Internal Medicine, Hallym University Kangdong Sacred Heart Hospital, Seoul, ${ }^{5}$ Regional Center for Respiratory Disease, Yeungnam University Medical Center, Yeungnam University College of Medicine, Daegu, ${ }^{6}$ Division of Pulmonary and Critical Care Medicine, Department of Internal Medicine, Soonchunhyang University Cheonan Hospital, Cheonan, ${ }^{7}$ Division of Pulmonary, Allergy and Critical Care Medicine, Department of Internal Medicine, Konkuk University School of Medicine, Seoul, ${ }^{8}$ Division of Pulmonary, Allergy and Critical Care Medicine, Department of Internal Medicine, Hallym University Medical Center, Hallym University College of Medicine, Anyang, Republic of Korea

Correspondence: Young Kyoon Kim; Chin Kook Rhee

Division of Pulmonary, Allergy and Critical Care Medicine, Department of Internal Medicine, Seoul St Mary's Hospital, College of Medicine, The Catholic University of Korea, 222 Banpodaero, Seochogu, Seoul 0659I, Republic of Korea

Tel +82 $222586059 ;+82222586067$

Fax +82 $25993589 ;+8225993589$

Email youngkim@catholic.ac.kr;

chinkook@catholic.ac.kr
Background: The chronic bronchitis (CB) phenotype has been associated with poor quality of life and an increased risk of disease in patients with COPD. However, little information exists regarding the relationship between the $\mathrm{CB}$ phenotype and the COPD assessment test (CAT) score. The goal of this study was to reveal the different pattern of CAT scores between $\mathrm{CB}$ and non-CB patients. Moreover, we aimed to investigate whether the $\mathrm{CB}$ phenotype is an independently associated factor for more symptom and high-risk groups.

Methods: Data were obtained from the Korea COPD Subgroup Study cohort recruited from 46 centers in South Korea since April 2012. CB patients were defined as having a chronic cough and sputum for 3 months per year, for a period of 2 consecutive years. We investigated the pattern of CAT and subquestionnaire scores between $\mathrm{CB}$ and non-CB patients. We also analyzed the proportion of $\mathrm{CB}$ phenotypes in each Global initiative for chronic Obstructive Lung Disease (GOLD) stage. Finally, we performed a logistic regression analysis to identify whether the $\mathrm{CB}$ phenotype was an independently associated factor for more symptom and high-risk groups

Results: Of the 1,106 study patients, $11.5 \%$ of patients were found to have a CB phenotype. CB phenotypes were most common in GOLD III (GOLD 2006) and GOLD D (GOLD 2015) stages. CAT scores were significantly higher in $\mathrm{CB}$ patients not only in terms of the total score but also for each subquestionnaire. Logistic regression revealed that the $\mathrm{CB}$ phenotype is an independently associated factor for more symptom and high-risk groups.

Conclusion: The present study revealed that $\mathrm{CB}$ patients have higher CAT scores and subquestionnaire results compared to non-CB patients. Additionally, we demonstrated that the $\mathrm{CB}$ phenotype is an independently associated factor for both more symptom and high-risk groups.

Keywords: chronic obstructive pulmonary disease, chronic bronchitis, COPD assessment test

\section{Introduction}

COPD is a major public health problem and is expected to be the third leading cause of death, and fifth leading disease burden, by $2020 .{ }^{1}$ In addition, its economic burden is reported to be substantial, especially when combined with chronic bronchitis (CB)..$^{2-4}$

Recent studies suggest that COPD is a heterogeneous disease and that different phenotypes may be associated with disease severity, quality of life, and mortality. ${ }^{5-8}$ $\mathrm{CB}$ is a common phenotype of COPD that has traditionally been considered to be on the opposite side of the disease spectrum to the emphysema phenotype. However, most individuals have both characteristics. ${ }^{2,9}$ The definition of CB has been variable but it is classically defined as the presence of a chronic cough and sputum for 3 months per year over a duration of 2 consecutive years. ${ }^{10,11}$ The prevalence of CB is reported to 
range from $14 \%$ to $74 \%$ of all patients with COPD, probably due to the varying definitions of $\mathrm{CB}$, different study populations, and different study designs. ${ }^{6,9,10}$

Previous investigations have revealed that the CB phenotype is associated with poor quality of life, as well as increased disease severity and risk of exacerbation in COPD patients. ${ }^{6,12-18}$ However, there has been a limited amount of data available regarding the relationship between the $\mathrm{CB}$ phenotype and the COPD assessment test (CAT) score. Since the CAT score was validated in 2009 , its usefulness in evaluating symptoms and quality of life has been emphasized. ${ }^{19,20}$ It is now an important factor in the new Global initiative for chronic Obstructive Lung Disease (GOLD) staging system as revised in $2015 .{ }^{21}$ Moreover, numerous studies have observed that the CAT score is associated with COPD severity and risk of exacerbation. ${ }^{22-25}$

In the GOLD 2015 staging system, COPD patients with a high CAT score $(\geq 10)$ or high modified Medical Research Council (mMRC) score ( $\geq 2$ ) are categorized into the more symptom group, and patients with a post-bronchodilator (BD) forced expiratory volume in 1 second $\left(\mathrm{FEV}_{1}\right)<50 \%$, or history of exacerbation $\geq 2 /$ year, or admission to hospital $\geq 1$ year are categorized into the high-risk group. The proportion of CB phenotypes in each GOLD stage has not yet been fully clarified.

The purpose of the present study was to identify the different characteristics between $\mathrm{CB}$ and non-CB COPD patients to determine whether the pattern of CAT scores varies between them. Moreover, we aimed to investigate whether the CB phenotype is an independently associated factor for the more symptom group (CAT score $\geq 10$ ). Additionally, we aimed to evaluate whether the $\mathrm{CB}$ phenotype is an independently associated factor for the high-risk group.

\section{Methods}

\section{Study design, study population, and data collection}

In this study, we used data from the KOCOSS cohort to investigate the difference in clinical outcomes between $\mathrm{CB}$ and non-CB patients. KOCOSS is an ongoing multicenter COPD cohort study that recruited participants from 47 centers in South Korea since April 2012. Approximately 1,565 patients were enrolled as of December 28, 2015. The inclusion criteria were Korean patients aged more than 40 years old and a ratio of $\mathrm{FEV}_{1}$ to forced vital capacity (FVC) of $<0.7$. Written informed consent was obtained from all the study patients. Ethics approval for this study was obtained from the ethics committee at each center. The names of ethics committees are as follows: Gacheon University Gil Medical Center, Hallym University Kangnam Sacred Heart Hospital, Gangnam Severance Hospital, Kyung Hee University Hospital at Gangdong, Hallym University Kangdong Sacred Heart Hospital, Kangbuk Samsung Hospital, Kangwon National University Hospital, Konkuk University Hospital, Konkuk University Chungju Hospital, Kyungpook National University Hospital, Gyeongsang National University Hospital, Korea University Guro Hospital, Korea University Anam Hospital, Seoul Eulji Hospital, Dongguk University Gyeongju Hospital, Dongguk University Ilsan Hospital, Keimyung University Dongsan Medical Center, Dong-A University Hospital, Hallym University Dongtan Sacred Heart Hospital, Pusan National University Hospital, Inje University Busan Paik Hospital, The Catholic University of Korea Bucheon St. Mary's Hospital, Soonchunhyang University Hospital Bucheon, Seoul National University Bundang Hospital, Bundang CHA Hospital, Seoul Metropolitan Government Seoul National University Boramae Medical Center, Samsung Medical Center, Soonchunhyang University Hospital Seoul, The Catholic University of Korea Seoul St. Mary's Hospital, The Catholic University of Korea St. Paul's Hospital, The Catholic University of Korea St. Vincent's Hospital, Severance Hospital, Asan Medical Center, Ajou University Hospital, The Catholic University of Korea Yeouido St. Mary's Hospital, The Catholic University of Korea Uijeongbu St. Mary’s Hospital, Yeungnam University Medical Center, Ulsan University Hospital, Wonkwang University Sanbon Hospital, Wonju Severance Christian Hospital, Ewha Womans University Mokding Hospital, Incheon St. Mary's Hospital, Inha University Hospital, Chonnam National University Hospital, Chonbuk National University Hospital, Jeju National University Hospital, Soonchunhyang University Hospital Cheonan, Hallym University Chuncheon Sacred Heart Hospital, Hallym University Sacred Heart Hospital, and Hanyang University Guri Hospital. We also received approval from each center to use their subjects' clinical records for the study while maintaining the confidentiality of the data.

CB was defined using the following self-administered questionnaires: 1) Do you experience a cough most days, for at least 3 months per year? 2) Have you had a cough for more than 2 consecutive years? 3) Do you produce sputum most days, for at least 3 months per year? 4) Have you had sputum for more than 2 consecutive years? If the patient answered "yes" to all questions, then the subject was classified as having CB. ${ }^{14}$ Patients who answered "I don't know" or did not answer a question were excluded from the study. 


\section{Clinical characterizations}

We evaluated the difference in symptoms and COPDrelated health status between $\mathrm{CB}$ and non-CB patients using the mMRC scale (range 0-4, higher scores mean worse), St George's Respiratory Questionnaire for COPD patients (SGRQ-C, range $0-100$, higher scores mean worse), and CAT score (range 0-40, higher scores mean worse). Specifically, we focused on the CAT score, which is the sum of eight items related to cough (CAT1), phlegm (CAT2), chest tightness (CAT3), breathlessness (CAT4), activity limitations (CAT5), confidence in leaving home (CAT6), sleep (CAT7), and energy (CAT8). The total CAT score was reviewed in addition to each item score to evaluate the symptoms and health status rather than just cough and sputum. The differences in age, sex, and pulmonary function test results of the two phenotypes were also analyzed. FEV ${ }_{1}$, CAT score, exacerbation, history of admission, and emergency room visitation were used to categorize patients into GOLD 2006 and revised GOLD 2015 stages to evaluate the prevalence of the CB phenotype in each stage. ${ }^{1,21} \mathrm{We}$ defined patients with a CAT score $\geq 10$ as the more symptom group and those with a CAT score $<10$ as the less symptom group. We also classified patients with a post-BD $\mathrm{FEV}_{1}<50 \%$, or a history of exacerbation $\geq 2 /$ year, or admission to hospital $\geq 1$ /year as the high-risk group and those without as the low-risk group.

\section{Statistical analysis}

Statistical analysis was performed with the SAS 9.3 (SAS Institute Inc., Cary, NC, USA) software. Quantitative variables are shown as mean \pm standard deviation, and categorical variables are reported as numbers and percentages. The total number in each analysis set is provided in Table 1. Categorical variables (eg, sex, current smoker, and CAT score $\geq 10$ ) were compared between groups using a chi-square test. Continuous variables (eg, age, body mass index, mMRC, SGRQ-C, CAT, post-BD FVC, post-BD $\mathrm{FEV}_{1}$, and post-BD $\mathrm{FEV}_{1} / \mathrm{FVC}$ ratio) were assessed using a Student's $t$-test. Differences in categorical variables (eg, the GOLD stage) were assessed using a Mantel-Haenszel test. A $P$-value of less than 0.05 was considered to be statistically significant.

A multiple logistic regression analysis was performed to assess the independent association of CB phenotype, sex, age, current smoking, and post-BD FEV ${ }_{1}$ on the more symptom group. To assess the independently associated factors for the high-risk group, we used a similar logistic regression model, excluding the post-BD FEV ${ }_{1}$ variable as it is a discriminating factor for both the high- and low-risk groups. Results from the logistic regression are presented with odds ratios (ORs) and 95\% confidence intervals (CIs). A Hosmer-Lemeshow test was conducted to assess the goodness of fit for the logistic regression models.

\section{Results \\ Baseline characteristics and prevalence of $\mathrm{CB}$}

A total of 1,148 patients were enrolled in the KOCOSS cohort study from April 2012 to May 2015. Forty patients were excluded due to missing data and two patients were excluded due to errors in the data set. Therefore, data analysis was performed on 1,106 subjects. The baseline characteristics of the study population are shown in Table 1 . The prevalence of CB was reported to be $11.5 \%(n=127)$. Male sex was predominant in both the non-CB and $\mathrm{CB}$ groups, and no significant difference was identified. The subjects in the non-CB group were significantly older than those in the $\mathrm{CB}$ group. The $\mathrm{CB}$

Table I Baseline characteristics of the COPD patients $(n=I, 106)$

\begin{tabular}{llll}
\hline Characteristics & Non-CB, $\mathbf{n = 9 7 9 ~ ( 8 8 . 5 \% ) ~}$ & CB, n=l27 (II.5\%) & P-value \\
\hline Sex (male, $\mathrm{n}[\%])$ & $973(91.6 \%)$ & $127(90.1 \%)$ & 0.70 \\
Age (years) & $70.9 \pm 7.8$ & $69.4 \pm 8.1$ & 0.05 \\
Current smoker (n [\%]) & $256(29.6 \%)$ & $43(39.45 \%)$ & 0.04 \\
BMI $\left(\mathrm{kg} / \mathrm{m}^{2}\right)$ & $22.8 \pm 3.4$ & $22.3 \pm 3.3$ & 0.13 \\
mMRC & $1.5 \pm 1.0$ & $1.9 \pm 1.0$ & $<0.01$ \\
SGRQ-C & $33.0 \pm 18.6$ & $46.1 \pm 22.0$ & $<0.01$ \\
CAT & $14.6 \pm 7.3$ & $21.2 \pm 8.2$ & $<0.01$ \\
Post-BD FVC (\% predicted) & $82.6 \pm 18.0$ & $82.5 \pm 17.9$ & 0.94 \\
Post-BD FEV (\% predicted) & $57.1 \pm 17.1$ & $51.4 \pm 13.7$ & $<0.01$ \\
Post-BD FEV $/$ FVC (\% predicted) & $49.5 \pm 12.1$ & $45.2 \pm 11.1$ & $<0.01$ \\
\hline
\end{tabular}

Notes: Data are expressed as number (\%) or mean \pm standard deviation. Categorical variables were compared between groups using a $\chi^{2}$ test. Continuous variables were assessed using a Student's $t$-test. A $P$-value of less than 0.05 was considered to be statistically significant.

Abbreviations: CB, chronic bronchitis; BMI, body mass index; mMRC, modified Medical Research Council; SGRQ-C, St George Respiratory Questionnaire for COPD patients; CAT, COPD assessment test; BD, bronchodilator; FVC, forced vital capacity; FEV , forced expiratory volume in I second. 


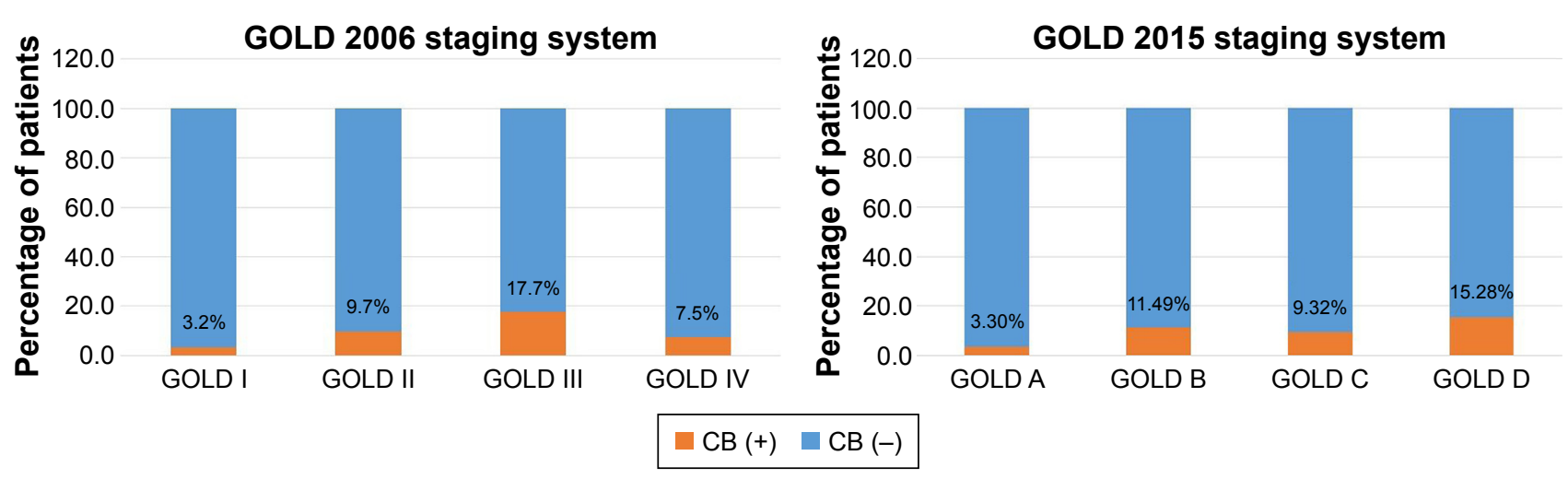

Figure I Percentage of patients with CB according to GOLD stage.

Abbreviations: GOLD, Global initiative for chronic Obstructive Lung Disease; $C B$, chronic bronchitis.

group had higher mMRC, SGRQ-C, and CAT scores, which indicated more symptoms and poorer COPD-related health status. The post-BD $\mathrm{FEV}_{1}$ and $\mathrm{FEV}_{1} / \mathrm{FVC}$ ratio were lower in the $\mathrm{CB}$ group and showed statistical significance. Among these statistically significant different variables, age difference was not clinically significant because it was minimal. Other variables (current smoker, mMRC, SGRQ-C, CAT, and lung function) showed clinically significant differences between the two groups. The percentage of $\mathrm{CB}$ according to GOLD stage is shown in Figure 1. With the GOLD 2006 staging system, CB was most common in GOLD III (17.7\%) and least common in GOLD I (3.2\%), with a $P$-value of 0.0309 . With the GOLD 2015 staging system, the CB phenotype was most common in GOLD D (15.28\%) and least common in GOLD A $(3.30 \%)$, with a $P$-value of 0.0001 .

\section{Comparison of CAT scores between CB and non-CB patients}

We compared the CAT scores between $\mathrm{CB}$ and non-CB patients. The total CAT score was statistically higher in the CB patients $(14.6 \pm 7.3$ vs $21.2 \pm 8.2, P<0.01)$. We also analyzed all eight items that comprise the CAT score questionnaire, and the scores of all items were found to be statistically higher in the CB patients (Figure 2). The CAT1 and CAT2 scores showed the greatest difference between $\mathrm{CB}$ and non-CB patients, and the CAT8 score showed the least (Table 2).

\section{Comparison between the more and less symptom groups}

The logistic regression for the more symptom group is shown in Table 3. The CB phenotype and post-BD FEV ${ }_{1}$ (\% predicted) were identified as independently associated factors for the more symptom group. The OR for the CB phenotype was 2.12 (95\% CI, 1.19-3.77).

\section{Comparison between the high- and low-risk groups}

The logistic regression for the high-risk group is shown in Table 4. In the regression model, the CB phenotype was

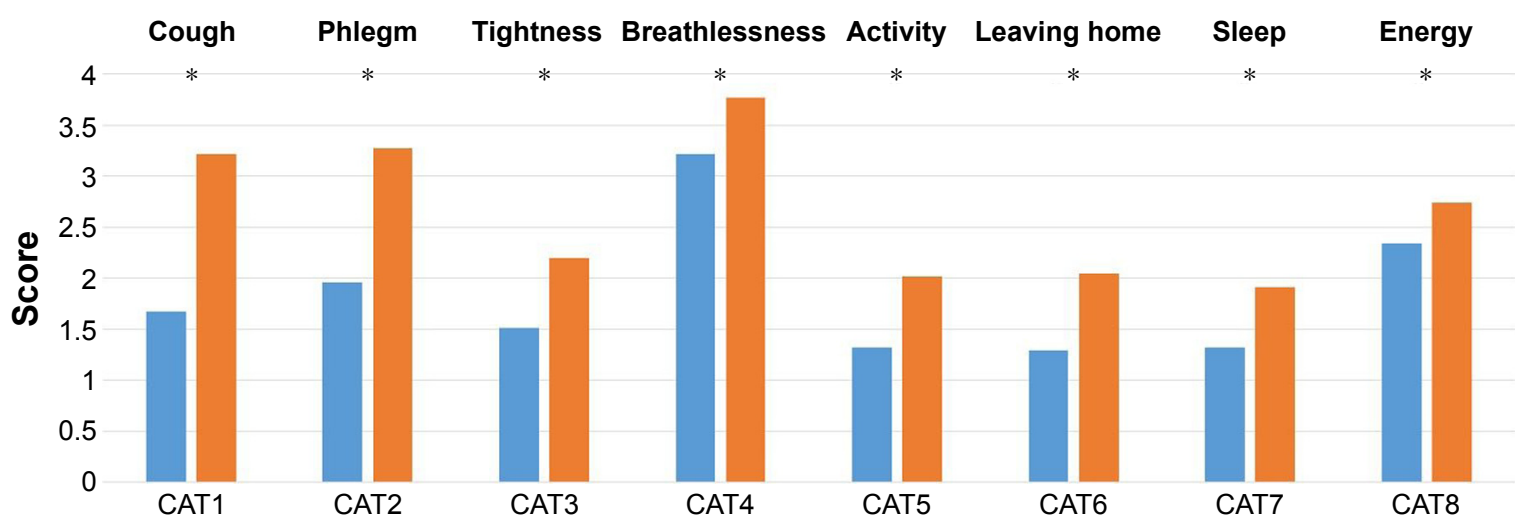

Figure 2 Comparison of $C A T$ scores between $C B$ and non- $C B$ patients.

Notes: $* P<0.05$. Blue color indicates $\mathrm{CB}(-)$, orange color indicates $\mathrm{CB}(+)$.

Abbreviations: $C A T, C O P D$ assessment test; $C B$, chronic bronchitis. 
Table 2 Comparison of each of the eight items of CAT scores between CB and non-CB patients

\begin{tabular}{|c|c|c|c|c|c|c|c|c|c|}
\hline & Total CAT & CATI & CAT2 & CAT3 & CAT4 & CAT5 & CAT6 & CAT7 & CAT8 \\
\hline CB $(-)$ & $14.62 \pm 7.30$ & $1.67 \pm 1.24$ & $1.96 \pm 1.29$ & $1.5 \mathrm{I} \pm 1.36$ & $3.22 \pm 1.33$ & $1.32 \pm 1.40$ & $1.29 \pm 1.39$ & $1.32 \pm 1.40$ & $2.34 \pm 1.24$ \\
\hline CB $(+)$ & $21.16 \pm 8.16$ & $3.22 \pm 1.32$ & $3.27 \pm 1.26$ & $2.20 \pm 1.53$ & $3.77 \pm 1.24$ & $2.02 \pm 1.48$ & $2.04 \pm 1.57$ & $\mid .91 \pm 1.51$ & $2.74 \pm 1.30$ \\
\hline Differences & 6.54 & 1.55 & 1.31 & 0.69 & 0.55 & 0.70 & 0.75 & 0.59 & 0.40 \\
\hline$P$-value & $<0.01$ & $<0.01$ & $<0.01$ & $<0.01$ & $<0.01$ & $<0.01$ & $<0.01$ & $<0.01$ & $<0.01$ \\
\hline
\end{tabular}

Notes: Data are expressed as mean \pm standard deviation. Variables were assessed using a Student's $t$-test. A P-value of less than 0.05 was considered to be statistically significant.

Abbreviations: CAT, COPD assessment test; $\mathrm{CB}$, chronic bronchitis.

shown to be an independently associated factor for the highrisk group. The OR for the CB phenotype was 1.55 (95\% CI, 1.03-2.34).

\section{Discussion}

In this large, multicenter, observational study, we found that the CAT score was higher in the CB group than in the non-CB group, even in terms of the subquestionnaires. It is evident from the definition that the $\mathrm{CB}$ group had more coughs (CAT1) and sputum (CAT2). However, the fact that other items (CAT3-8) were also significantly higher in the $\mathrm{CB}$ group is of note. This indicates that $\mathrm{CB}$ patients have more symptoms, such as breathlessness and chest tightness, and a poor COPD-related health status, such as confidence in leaving home, activity limitations, sleep, and energy levels. High CAT score in the CB group may be partially because of $\mathrm{CB}$ definition. However, despite that, SGRQ-C, lung function, and $\mathrm{mMRC}$ were also significantly different between $\mathrm{CB}$ and non-CB groups. This result suggests that high $\mathrm{CAT}$ score in the $\mathrm{CB}$ group represents high disease burden and poor quality of life in these patients.

We divided the patients with GOLD 2006 and GOLD 2015 stages into separate groups and analyzed the prevalence of CB in each group. Previous studies have shown that the proportion of the $\mathrm{CB}$ phenotype increases with GOLD 2006 stage. ${ }^{6,713}$ However, in this study, the proportion of CB phenotype increased from GOLD I to III but the correlation was reversed in GOLD IV group. Moreover, with the GOLD 2015 staging system, we found that CB was most prevalent in GOLD D (15.28\%), followed by GOLD B (11.49\%). This

Table 3 Multiple logistic regression analysis of the associated factors for the more symptom group

\begin{tabular}{lll}
\hline Variable & OR $(\mathbf{9 5 \%} \mathbf{C I})$ & P-value \\
\hline CB phenotype & $2.12(1.19-3.77)$ & 0.01 \\
Sex (male) & $0.93(0.42-2.05)$ & 0.86 \\
Age & $1.02(1.00-1.04)$ & 0.11 \\
Current smoker & $1.03(0.74-1.44)$ & 0.87 \\
Post-BD FEV, (\% predicted) & $0.97(0.97-0.98)$ & $<0.01$ \\
\hline
\end{tabular}

Notes: Hosmer-Lemeshow goodness of fit; $P=0.89$.

Abbreviations: $\mathrm{OR}$, odds ratio; $\mathrm{Cl}$, confidence interval; $\mathrm{CB}$, chronic bronchitis; $\mathrm{BD}$, bronchodilator; $\mathrm{FEV}_{1}$, forced expiratory volume in I second. may be associated with a high CAT score in the GOLD B and D stages, by definition. A previous study by Han et al as part of the COPDGene study reported similar results, but in this case CB was more prevalent in GOLD B than in GOLD D $(35 \%$ vs $32.7 \%){ }^{26}$

In this study, we determined that the CB phenotype was an independent factor for the more symptom group. Recent studies have demonstrated that the $\mathrm{CB}$ group has poor symptoms and quality of life, but these studies were mostly based on other clinical indices such as the mMRC or SGRQ scores, unlike our study. ${ }^{7,13,15-18,27}$ In the ECLIPSE study, Agusti et al reported significant differences in $\mathrm{MMRC}$ and SGRQ scores between the $\mathrm{CB}$ and non-CB groups in an overall group of COPD patients $(P<0.0001)$, but these did not correspond to each GOLD subgroup. ${ }^{7}$ However, differences in the SGRQ scale were statistically significant in all GOLD groups. In the COPDGene study, Kim et al found that the CB group was more symptomatic than the non-CB group according to the mMRC ( $2.55 \pm 1.31$ vs $2.11 \pm 1.41, P<0.0001)$ and SGRQ (49.9 \pm 19.7 vs $36.6 \pm 20.0, P<0.0001)$ scales. ${ }^{17}$ In the PLATINO study, de Oca et al assessed the CB phenotype using multivariate analysis and concluded that the $\mathrm{CB}$ phenotype was associated with additional symptoms such as wheezing (OR 2.40) and dyspnea (OR 2.42), and also with a poor general health status assessed by the Short Form-12 generic quality of life questionnaire (OR 0.60$).{ }^{13}$

Previous studies have shown that the $\mathrm{CB}$ phenotype is associated with a high rate of exacerbation and poor lung function. ${ }^{6,9,15,17}$ In addition, some reports have indicated that the $\mathrm{CB}$ phenotype is associated with frequent hospitalization. ${ }^{28}$

Table 4 Multiple logistic regression analysis of associated factors for the high-risk group

\begin{tabular}{lll}
\hline Variable & OR (95\% CI) & P-value \\
\hline CB phenotype & I.55 (I.03-2.34) & 0.04 \\
Sex (male) & $1.40(0.72-2.72)$ & 0.33 \\
Age & $1.00(0.98-1.02)$ & 0.97 \\
Current smoker & $0.80(0.60-1.05)$ & 0.04 \\
\hline
\end{tabular}

Notes: Hosmer-Lemeshow goodness of fit; $P=0.30$.

Abbreviations: $\mathrm{OR}$, odds ratio; $\mathrm{Cl}$, confidence interval; $\mathrm{CB}$, chronic bronchitis. 
The results of the present study correspond well with those of earlier studies. We performed multivariate analysis to prove that the $\mathrm{CB}$ phenotype is an independent risk factor for the high-risk group.

In this study, $\mathrm{CB}$ was prevalent in $11.5 \%$ of the COPD patients. This result is relatively lower than previous studies, which reported a prevalence of $14 \%-74 \%{ }^{6,9}$ Considering that the prevalence of $\mathrm{CB}$ in clinical-based studies was higher (27.3\%-74.1\%) than population-based studies (14\%-30\%), our clinical study showed a much lower prevalence than previous studies. ${ }^{6}$ However, because the definition of $\mathrm{CB}$ is variable, our definition using chronic cough and sputum may have lowered the prevalence compared with other studies that defined CB with chronic sputum only. ${ }^{10,13,29,30}$ Additionally, the definition of CB can be ambiguous when translated into Korean, especially the phrase " 2 consecutive years". Therefore, patients may not have understood the questionnaire, and this may have caused a reduction in the response rate.

Our study has several limitations. First, subjects in the study were recruited from populations who were receiving treatment from the participating hospitals - these hospitals are mainly tertiary medical centers. Therefore, our results may not be consistent with the general population. Second, this was a cross-sectional study. In this study, we did not use follow-up data relating to exacerbation. Although prospective follow-up data for exacerbation exist for this cohort, the data were insufficient because the cohort is relatively new and therefore the follow-up period was short in most cases. When we classified the high- and low-risk groups, we used a simple question relating to the previous history of exacerbation over 1 year. Thus, there is the potential for some recall bias, and the history of exacerbation may have been underestimated in some patients. Third, there is a possibility of misclassification of $\mathrm{CB}$ patients in this study given the self-report status and potential misunderstanding of the question in Korean. This may underestimate the prevalence of $\mathrm{CB}$ in this cohort. As a result, among real $\mathrm{CB}$ patients, only some more symptomatic $\mathrm{CB}$ patients may be classified as $\mathrm{CB}$ group in this study. This may have affected the result of this study.

\section{Conclusion}

In summary, our data support the fact that $\mathrm{CB}$ patients have a higher CAT score than non-CB patients. This is consistent not only in terms of the total CAT score but also for each subquestionnaire in addition to those querying cough and sputum. Moreover, this study revealed that the $\mathrm{CB}$ phenotype is an independent risk factor for both the more symptom and high-risk groups, as defined by GOLD 2015 guidelines.

\section{Disclosure}

The authors report no conflicts of interest in this work.

\section{References}

1. Rabe KF, Hurd S, Anzueto A, et al. Global strategy for the diagnosis, management, and prevention of chronic obstructive pulmonary disease. Am J Respir Crit Care Med. 2007;176(6):532-555.

2. AbuDagga A, Sun SX, Tan H, Solem CT. Healthcare utilization and costs among chronic bronchitis patients treated with maintenance medications from a US managed care population. J Med Econ. 2013; 16(3):421-429.

3. Blanchette CM, Roberts MH, Petersen H, Dalal AA, Mapel DW. Economic burden of chronic bronchitis in the United States: a retrospective case-control study. Int J Chron Obstruct Pulmon Dis. 2011;6:73-81.

4. Pasquale MK, Sun SX, Song F, Hartnett HJ, Stemkowski SA. Impact of exacerbations on health care cost and resource utilization in chronic obstructive pulmonary disease patients with chronic bronchitis from a predominantly Medicare population. Int J Chron Obstruct Pulmon Dis. 2012;7:757-764.

5. Rhee CK. Phenotype of asthma-chronic obstructive pulmonary disease overlap syndrome. Korean J Intern Med. 2015;30(4):443-449.

6. Corhay JL, Vincken W, Schlesser M, Bossuyt P, Imschoot J. Chronic bronchitis in COPD patients is associated with increased risk of exacerbations: a cross-sectional multicentre study. Int J Clin Pract. 2013; 67(12):1294-1301

7. Agusti A, Calverley PM, Celli B, et al. Characterisation of COPD heterogeneity in the ECLIPSE cohort. Respir Res. 2010;11:122.

8. Agusti A. Phenotypes and disease characterization in chronic obstructive pulmonary disease. Toward the extinction of phenotypes? Ann Am Thoracic Soc. 2013;10(Suppl):S125-S130.

9. Kim V, Criner GJ. Chronic bronchitis and chronic obstructive pulmonary disease. Am J Respir Crit Care Med. 2013;187(3):228-237.

10. Burgel PR. Chronic cough and sputum production: a clinical COPD phenotype? Eur Respir J. 2012;40(1):4-6.

11. Mannino DM. Chronic obstructive pulmonary disease: definition and epidemiology. Respir Care. 2003;48(12):1185-1191; discussion 1191-1183

12. Allen-Ramey FC, Gupta S, DiBonaventura MD. Patient characteristics, treatment patterns, and health outcomes among COPD phenotypes. Int J Chron Obstruct Pulmon Dis. 2012;7:779-787.

13. de Oca MM, Halbert RJ, Lopez MV, et al. The chronic bronchitis phenotype in subjects with and without COPD: the PLATINO study. Eur Respir J. 2012;40(1):28-36.

14. Kanervisto M, Saarelainen S, Vasankari T, et al. COPD, chronic bronchitis and capacity for day-to-day activities: negative impact of illness on the health-related quality of life. Chronic Respir Dis. 2010; 7(4):207-215

15. Kim V, Criner GJ. The chronic bronchitis phenotype in chronic obstructive pulmonary disease: features and implications. Curr Opin Pulm Med. 2015;21(2):133-141.

16. Kim V, Davey A, Comellas AP, et al. Clinical and computed tomographic predictors of chronic bronchitis in COPD: a cross sectional analysis of the COPDGene study. Respir Res. 2014;15:52.

17. Kim V, Han MK, Vance GB, et al. The chronic bronchitic phenotype of COPD: an analysis of the COPDGene Study. Chest. 2011;140(3): 626-633.

18. Ramos FL, Krahnke JS, Kim V. Clinical issues of mucus accumulation in COPD. Int J Chron Obstruct Pulmon Dis. 2014;9:139-150.

19. Becker C, Schafer J, Carvalho LL, Vitiello IP, da Silva AL. CAT correlates positively with respiratory rate and is a significant predictor of the impact of COPD on daily life of patients: a cross sectional study. Multidiscip Respir Med. 2014;9(1):47. 
20. Jones PW, Harding G, Berry P, Wiklund I, Chen WH, Kline Leidy N. Development and first validation of the COPD Assessment Test. Eur Respir J. 2009;34(3):648-654.

21. Fu JJ, McDonald VM, Gibson PG, Simpson JL. Systemic inflammation in older adults with asthma - COPD overlap syndrome. Allergy Asthma Immunol Res. 2014;6(4):316-324.

22. Ladeira I, Gomes T, Castro A, Ribeiro C, Guimaraes M, Taveira N. The overall impact of COPD (CAT) and BODE index on COPD male patients: correlation? Rev Port Pneumol. 2015;21(1):11-15.

23. Lee SD, Huang MS, Kang J, et al. The COPD assessment test (CAT) assists prediction of COPD exacerbations in high-risk patients. Respir Med. 2014;108(4):600-608.

24. Mackay AJ, Donaldson GC, Patel AR, Jones PW, Hurst JR, Wedzicha JA. Usefulness of the chronic obstructive pulmonary Disease Assessment Test to evaluate severity of COPD exacerbations. Am J Respir Crit Care Med. 2012;185(11):1218-1224.

25. Varol Y, Ozacar R, Balci G, Usta L, Taymaz Z. Assessing the effectiveness of the COPD Assessment Test (CAT) to evaluate COPD severity and exacerbation rates. COPD. 2014;11(2):221-225.
26. Han MK, Muellerova H, Curran-Everett D, et al. GOLD 2011 disease severity classification in COPDGene: a prospective cohort study. Lancet Respir Med. 2013;1(1):43-50.

27. Ferre A, Fuhrman C, Zureik M, et al. Chronic bronchitis in the general population: influence of age, gender and socio-economic conditions. Respir Med. 2012;106(3):467-471.

28. Vestbo J, Prescott E, Lange P. Association of chronic mucus hypersecretion with FEV1 decline and chronic obstructive pulmonary disease morbidity. Copenhagen City Heart Study Group. Am J Respir Crit Care Med. 1996;153(5):1530-1535.

29. Prescott E, Lange P, Vestbo J. Chronic mucus hypersecretion in COPD and death from pulmonary infection. Eur Respir J. 1995;8(8): $1333-1338$.

30. Miravitlles M, Guerrero T, Mayordomo C, Sanchez-Agudo L, Nicolau F, Segu JL. Factors associated with increased risk of exacerbation and hospital admission in a cohort of ambulatory COPD patients: a multiple logistic regression analysis. The EOLO Study Group. Respir Int Rev Thoracic Dis. 2000;67(5):495-501.
International Journal of COPD

\section{Publish your work in this journal}

The International Journal of COPD is an international, peer-reviewed journal of therapeutics and pharmacology focusing on concise rapid reporting of clinical studies and reviews in COPD. Special focus is given to the pathophysiological processes underlying the disease, intervention programs, patient focused education, and self management protocols.

\section{Dovepress}

This journal is indexed on PubMed Central, MedLine and CAS. The manuscript management system is completely online and includes a very quick and fair peer-review system, which is all easy to use. Visit http://www.dovepress.com/testimonials.php to read real quotes from published authors.

Submit your manuscript here: http://www.dovepress.com/international-journal-of-chronic-obstructive-pulmonary-disease-journal 